\title{
Trumping science
}

\author{
The scientific integrity of US government agencies is under attack from the Trump administration. Agency senior \\ executives and industry leaders need to continue pushing back.
}

A $t$ the beginning of September, under looming pressure from the White House to rush vaccine approval before November's election, eight US Food and Drug Administration (FDA) executives wrote a national newspaper column reiterating their commitment to "decisions guided by science." The same week, nine vaccine manufacturers also announced a pledge to abide by a "rigorous scientific and regulatory process" as they work toward potential global regulatory filings. This follows four former US Centers of Disease Control (CDC) directors warning of political appointees' "repeated efforts to subvert sound public health guidelines." Such moves are unprecedented, sounding the alarm that scientific integrity of US government agencies is being threatened by the Trump administration. In the coming months, senior government researchers and industry representatives must continue to speak out in defense of agency independence, transparency and regulatory norms. Letting political rhetoric and machinations unduly influence decisions will compromise public trust not only in federal agencies' credibility, but also in the products they regulate.

President Donald Trump has never had much affinity for science-based policy or for following norms. But with electoral prospects linked to rapid economic recovery after lockdown, his attacks on the independence of federal agencies are coming thicker and faster. Tweets accuse the FDA of being "the deep state" and delaying COVID-19 vaccines and drugs; speeches tout dubious drugs and muse on household disinfectants as cures; science-based advice on personal protective equipment, diagnostic testing and school reopening from the CDC is belittled, blocked or doctored.

Political interference in US federal agencies is nothing new: the Reagan, Bush and Obama administrations all meddled to various degrees. What is different today is the sheer scale and scope of the Trump administration's bullying, manipulation and lies.

In response to COVID-19, the White House has deprioritized input from experts, marginalized the CDC and restricted the ability of its scientists to speak directly to the public or media, as well as attempted to conceal data that is in the public interest. According to former Biomedical Advanced Research and Development Authority (BARDA) chief Rick Bright, cronyism and political meddling are now endemic at the Department of Health and Human Services.

The CDC's voice on COVID-19, already hushed, is being stifled. During the first 13 weeks of the outbreak - with 776,093 US infections and 41,758 deaths - it held only 19 briefings. In contrast, for SARS - a disease that had infected 8 Americans and killed none - it held 18 briefings.

As the election approaches, the situation is worsening.

CDC briefed the media just once during August. Administration officials have started to change, delay and prevent agency reports that are perceived as damaging to the president's optimistic pandemic messaging. For the first time since 1878, the CDC's Morbidity and Mortality Weekly Report is now subject to approval by political appointees.

At the end of August, pressure on FDA hierarchy for a positive spin on data became all too clear. In a presidential press briefing on the Emergency Use Authorization (EUA) of convalescent plasma for COVID-19, FDA Commissioner Stephen Hahn made misleading statements about the treatment's efficacy, which he later walked back in a tweet. Extraordinarily, the FDA press release headline announcing the plasma EUA stated it was "another achievement in administration's fight against pandemic."

All of this matters because the Trump administration's science denialism and interference has hobbled the country's COVID-19 response, undermined agency morale and ability to attract talent, and poisoned public perception of these government agencies. According to a September tracking poll by the Kaiser Family Foundation, four in ten adults say the FDA (39\%) and the CDC (42\%) are paying "too much attention" to politics when it comes to reviewing and approving coronavirus treatments and issuing guidelines and recommendations. Trust in the CDC has fallen by 16 percentage points since April.

The president's misinformation blitz also underscores the importance of transparent and rigorous FDA review of the safety and efficacy of COVID-19 vaccines and therapies. This should include disclosing clinical data and information underlying any decision to approve or authorize a COVID-19 vaccine following the advisory committee meeting on 22 October. Trust will be built if established processes are followed; it will be eroded if there are departures from the norm.

In addition, the presidential press briefings for EUAs on hydroxychloroquine and convalescent plasma illustrate that the White House is no place for FDA officials to discuss complex regulatory decisions. Such proceedings, which are dominated by the president's rhetoric, make it difficult to convey accurate information to press and public.

Officials at the CDC and elsewhere must continue to speak out and resist the doctoring and censorship of their reports and the scientific data they collect and distribute. Keeping as much information as possible in the public domain and out of the control of politically appointed officials will support science-based decision-making and enable the self-correction of errors.

The public stand taken by senior FDA executives and former CDC directors and the commitment from the nine biopharma CEOs to uphold the integrity of the scientific process are commendable. Companies should also share trial data in peer-reviewed publications and provide full disclosure of clinical study protocols for phase 3 trials, which some have now started to do.

Even under the best circumstances, public health and regulatory decision-making is a messy combination of law, policy, data, opinion and political context. But in today's hyperpolarized environment, the mix is being skewed towards politics and away from data. It is the duty of any US president to maintain the credibility and integrity of US regulatory agencies by supporting their transparency and independence. Without access to evidence and the reasoning behind decisions, the public will likely assume the worst. For now, the FDA, CDC and biopharma industry must provide the clarity the moment demands.

Published online: 24 September 2020 https://doi.org/10.1038/s41587-020-0713-y 